\title{
Jogos Educacionais como Ferramenta de Auxílio ao Processo de Alfabetização
}

\author{
Sivaldo Joaquim de Santana ${ }^{1}$, Wilk Oliveira ${ }^{2}$ \\ ${ }^{1}$ Núcleo de Excelência em Tecnologias Sociais (NEES) - Instituto de Computação (IC) \\ Universidade Federal de Alagoas (UFAL) - CEP: 57.072-900 - Maceió, AL - Brasil. \\ ${ }^{2}$ Laboratório de Computação Aplicada à Educação e Tecnologia Social Avançada \\ (CAEd) - Instituto de Ciências Matemáticas e de Computação (ICMC) \\ Universidade de São Paulo (USP) - CEP: 13.566-590 - São Carlos, SP - Brasil. \\ sivaldojoaquimeic.ufal.br, wilk.oliveira@usp.br
}

Resumo. Apesar do interesse dos docentes de diferentes niveis educacionais em utilizar jogos digitais para potencializar os processos de ensino e aprendizagem, o uso efetivo desses recursos nos anos iniciais do Ensino Fundamental I ainda é um grande desafio. Esse artigo tem por objetivo relatar a experiência da utilização de jogos digitais educativos no processo de alfabetização de 87 estudantes com idade entre 6 e 7 anos em uma escola de Educação Básica Brasileira, visando verificar o impacto desses jogos no desempenho dos estudantes. Para isso, foi utilizada uma abordagem qualitativa com ênfase na observação participante como técnica de coleta de dados. Como resultados, verificou-se que o uso de jogos digitais educativos aumentou a motivação, engajamento, atenção e impactou positivamente no desempenho de aprendizagem dos estudantes.

\begin{abstract}
Despite the interest of teachers and instructors of different educational levels in using digital games to enhance the teaching and learning processes, the effective use of these resources in the Elementary School is still a great challenge. This paper aims to report the experience of the use of digital educational games in the process of literacy of 87 students, with age between 6 and 7 years old, in a Brazilian Basic Education school, in order to verify the impact of these games on student performance. For this, a qualitative approach was used, with emphasis on participant observation as a data collection technique. As results, it was verified that the use of digital educational games increased the student's motivation, engagement, attention and positively impacted in their learning performance.
\end{abstract}

\section{Introdução}

Na atualidade, o desenvolvimento e utilização das Tecnologias Digitais de Informação e Comunicação (TDIC) tem transformado o modo como as pessoas e instituições se comunicam e desenvolvem seus produtos e/ou serviços [Brasil 2018b]. Na Educação Básica, existe uma tendência por parte da comunidade científica para a construção, aplicação, avalição e utilização das TDIC visando potencializar os processos de ensino e de aprendizagem dos estudantes [Pedró 2016; Santana e Santos 2018a; Santana e Santos 2018b; Santos e Isotani 2018]. 
VIII Congresso Brasileiro de Informática na Educação (CBIE 2019)

Anais do XXV Workshop de Informática na Escola (WIE 2019)

No Brasil, as políticas públicas desenvolvidas pelo Ministério da Educação (MEC) e discutidas com a sociedade, comunidade acadêmica e científica têm contribuído para a inserção das TDIC no ambiente educacional [Inep 2015; Brasil 2017; Brasil 2018a; Educação Conectada 2017]. Além disso, estudos recentes têm apresentado impactos positivos da aprendizagem dos estudantes com uso das TDIC como ferramentas para auxiliar nos processos de ensino e aprendizagem nas etapas da Educação Infantil [Santos e Silva Junior 2016; Santana e Santos 2018a; Santana e Santos 2018b], Ensino Fundamental [Santana et al. 2016; Vidotto et al. 2017; Santana et al. 2018; Neto et al. 2018] e no Ensino Médio [Santos et al. 2017; Bordin e Quepfert 2018; Oliveira 2018]. Entretanto, nota-se que o uso das TDIC na sala de aula com estudantes dos anos iniciais do Ensino Fundamental I ainda é um desafio no cenário brasileiro.

Visto que, na prática, em função da dificuldade de aplicação e avaliação de estudos nesse cenário, um número reduzido de trabalhos foram conduzidos e, por conseguinte, poucos resultados efetivos relacionados ao uso de TDIC com estudantes dessa faixa etária são sabidos, chamando a atenção para necessidade de novos estudos que abordem a seleção, uso e o impacto na aprendizagem com a utilização dos recursos digitais em ambiente do mundo real, como destacado nos desafios da Organização das Nações Unidas para a Educação, a Ciência e a Cultura [Unesco 2017] e pela própria Base Nacional Comum Curricular (BNCC) [Brasil 2018a], que para a Educação Infantil e o Ensino Fundamental, entre as dez competências gerais, visa desenvolver habilidades como "explorar diferentes tecnologias e recursos digitais (multimeios, animações, jogos eletrônicos, gravações em áudio e vídeo, fotografia, softwares, etc) nos processos de criação artística" [Brasil 2018a, p. 203].

Diante desse desafio, o presente artigo relata a experiência de uso das TDIC nos anos iniciais da Educação Básica, por meio da análise do impacto dos jogos digitais educativos no processo de alfabetização dos estudantes no $1^{\circ}$ ano e $2^{\circ}$ ano do Ensino Fundamental I. Para isso, foram utilizados dois jogos digitais, denominados: "Capitão Alberto e o Tesouro do Faraól" e "Chapeuzinho e o Enigma da Floresta" " disponíveis online e ambos gratuitos, com um total de $N=87$ estudantes, na faixa etária de 6 e 7 anos de idade, em uma escola de Educação Infantil, Ensino Fundamental e Médio, localizado ao Sul do Estado de Alagoas. Neste trabalho, foi utilizada como método de pesquisa, a abordagem qualitativa com ênfase na observação participante como técnica de coleta de dados.

Os principais resultados obtidos nesse estudo revelam que o uso adequado das TDIC alinhados aos processos de ensino e aprendizagem impactou positivamente no desempenho dos estudantes, despertou a atenção, aumentou à motivação, o engajamento no desenvolvimento das atividades por meio dos jogos digitais educativos e disseminou o trabalho colaborativo entre os estudantes nos processos de ensino e aprendizagem no laboratório de informática. Além disso, o uso dos jogos ampliou o diálogo entre os estudantes e professores no processo de alfabetização. Esses resultados contribuem no desenvolvimento cognitivo dos estudantes, para inserção e uso da TDIC na escola, para a elaboração de políticas públicas e recursos digitais educacionais com foco na aprendizagem significativa, prazerosa, de qualidade e inclusiva.

\footnotetext{
${ }^{1}$ Disponível em: http://brincandocomarie.com.br/capitao-alberto-farao/

${ }^{2}$ Disponível em: http://brincandocomarie.com.br/chapeuzinho-enigma/
} 
VIII Congresso Brasileiro de Informática na Educação (CBIE 2019)

Anais do XXV Workshop de Informática na Escola (WIE 2019)

Este artigo apresenta a seguinte estrutura: na sequência, apresentamos na seção 2 alguns trabalhos relacionados que deram embasamento teórico para a condução deste estudo; na seção 3 dissertamos a respeito dos materiais e métodos utilizados na realização desta pesquisa; na seção 4 mostramos os resultados obtidos, e por fim, na seção 5 apresentamos as considerações finais do presente estudo.

\section{Trabalhos Relacionados}

O desenvolvimento, utilização e avaliação de recursos tecnológicos como ferramenta para apoiar estudantes e professores nos processos de ensino e aprendizagem em diferentes domínios e níveis no contexto educacional tem sido objeto de estudo de vários pesquisadores [Oliveira e Silva Junior 2015; Sousa Pires et al. 2018; Santana e Santos 2018a; Santana e Santos 2018b; Santos J. et al. 2018; Oliveira et al. 2019].

Neste contexto, Ramos e Segundo (2018) conduziram um estudo do tipo quaseexperimental com abordagem quantitativa envolvendo 100 estudantes do $2^{\circ}$ ano e $3^{\circ}$ ano do Ensino Fundamental, com idades entre 7 e 9 anos, divididos em dois grupos: experimental (com o uso de jogos) e controle (sem o uso de tecnologia). Com objetivo de avaliar os efeitos do uso de jogos digitais no contexto escolar para o aprimoramento da atenção e da flexibilidade cognitiva. Os resultados revelaram que o grupo experimental em comparação ao controle teve uma melhora significativa em relação à atenção $(p<0,005)$ e a flexibilidade cognitiva $(p<0,05)$, sugerindo que o uso de jogos digitais pode contribuir com o aprimoramento das funções executivas.

No trabalho de Morais et al. (2018) relata-se a experiência com o uso de jogos digitais como ferramenta para auxiliar no processo de alfabetização de 50 estudantes do $1^{\circ}$ ano do Ensino Fundamental em uma escola pública municipal, situada na cidade de Petrolina, no estado de Pernambuco. O estudo de natureza explicativa utilizou cinco jogos educativos gratuitos online: "Ludo Educativo - Primeiros Passos", "Fabrica de Palavras", "Forma Palavras", "Ordene as frases" ${ }^{6 "}$ e "Histórias Fantásticas"”, visando promover a leitura e a escrita na alfabetização. Como resultados, observou-se que o uso de jogos digitais despertou o interesse e motivação dos estudantes em aprender de forma lúdica, interativa e prazerosa, contribuindo assim, para uma aprendizagem mais significativa.

Recentemente, Ramos et al. (2018) conduziu um estudo com utilização de jogos digitais que integra a "Escola do Cérebro" com estudantes da Educação Básica visando compreender a percepção dos estudantes quanto à sua motivação com relação à intervenção proposta, bem como o desenvolvimento de suas habilidades cognitivas. Para isso, foi realizada uma pesquisa de campo com abordagem qualitativa envolvendo 25 estudantes do $2^{\circ}$ ano do Ensino Fundamental I, com uma média de 8 anos de idade. De acordo com os mesmos pesquisadores, os resultados revelaram que os estudantes apresentaram um alto nível de motivação. Entretanto, não foi identificada uma

\footnotetext{
${ }^{3}$ Disponível em: https://www.ludoeducativo.com.br/pt/play/ludo-primeiros-passos

${ }^{4}$ Disponível em: http://www.escolagames.com.br/jogos/fabricaPalavras/

${ }^{5}$ Disponível em: http://www.escolagames.com.br/jogos/formaPalavras/

${ }^{6}$ Disponível em: https://www.smartkids.com.br/jogos-educativos/jogo-ordene-as-frases/

${ }^{7}$ Disponível em: http://www.proativa.virtual.ufc.br/objetos_aprendizagem/historias/

${ }^{8}$ Disponível em: $\underline{\text { https://escoladocerebro.com/pt/home-pt/ }}$
} 
VIII Congresso Brasileiro de Informática na Educação (CBIE 2019)

Anais do XXV Workshop de Informática na Escola (WIE 2019)

associação significativa $(p>0,05)$ entre o nível de motivação e habilidades cognitivas (atenção e resolução de problemas).

Diante dos estudos mencionados, apesar do crescente interesse no desenvolvimento e utilização de jogos digitais educativos pela comunidade acadêmica visando aumentar o engajamento, a motivação e melhorar o desempenho de aprendizagem dos estudantes do Ensino Fundamental [Ramos e Segundo 2018; Morais, Castro e Santos 2018], a sua aplicação de forma efetiva no contexto educacional ainda é um desafio para os docentes no cenário brasileiro [Bergamasco E. \& Bergamasco L. 2013] por diversos fatores, entre eles, a falta de infraestrutura física, suporte tecnológica e a necessidade de formação continuada dos docentes para o uso adequado dos recursos digitais alinhados aos objetivos e processos didático-pedagógicos [Figueiredo, Nobre e Passos 2015]. Diante disto, fica evidente a necessidade de conduzir novos estudos que aborde o uso de jogos educacionais como ferramenta de auxílio ao processo de ensino e aprendizagem [Ramos et al. 2018] em cenário do mundo real. Além disso, promove a inclusão da cultura digital dos estudantes através de práticas pedagógicas de forma lúdica com o uso das TDIC nos anos iniciais do Ensino Fundamental visando o desenvolvimento das competências e habilidades da BNCC.

\section{Materiais e Métodos}

O presente estudo foi realizado com 5 turmas dos anos iniciais do Ensino Fundamental I, conforme mostrado na Tabela 1 , com um total de $N=87$, sendo 36 estudantes do sexo masculino e 51 do sexo feminino, na faixa etária de 6 e 7 anos de idade, fazendo uso de dois jogos educativos digitais, em uma escola de Educação Infantil, Ensino Fundamental e Médio, situada na região do Baixo do São Francisco, localizado ao Sul do Estado de Alagoas. Vale ressaltar, que o número de estudantes mostrado na Tabela 1 corresponde ao total de cada turma. $\mathrm{O}$ fato de existir apenas 6 estudantes participantes no $1^{\circ}$ Ano "C" ocorre em função da preferência dos pais da região em matricular os estudantes nessa faixa etária e ano escolar no período matutino.

Tabela 1. Relação de turmas e número de estudantes participantes

\begin{tabular}{|c|c|c|c|c|}
\hline \multirow{2}{*}{ Nível } & \multirow{2}{*}{ Ano/Turma } & \multicolumn{2}{|c|}{ Gêneros } & \multirow{2}{*}{$\begin{array}{c}\text { Número de Estudantes } \\
\text { por Turma }\end{array}$} \\
\hline & & Masculino & Feminino & \\
\hline \multirow{6}{*}{ 。䏤 } & $1^{\circ}$ Ano "A" & 7 & 12 & 19 \\
\hline & $1^{\circ}$ Ano "B" & 6 & 11 & 17 \\
\hline & $1^{\circ}$ Ano "C" & 3 & 3 & 6 \\
\hline & $2^{\circ}$ Ano "A" & 10 & 14 & 24 \\
\hline & $2^{\circ}$ Ano "B" & 10 & 11 & 21 \\
\hline & \multicolumn{3}{|c|}{ Total de estudantes } & 87 \\
\hline
\end{tabular}

Os jogos utilizados fazem parte de uma plataforma web gratuita, denominada "Brincando com Ariê" processo de alfabetização de estudantes da Educação Básica. A plataforma visa estimular o aprendizado por meio de atividades com foco na qualidade de design e usabilidade de jogos educativos [Brincando com Arie 2019]. Dentro do ambiente, o estudante pode visualizar todos os jogos ou selecionar as atividades educativas através das categorias: Brincando com Ariê, Português, Matemática e Idiomas.

\footnotetext{
${ }^{9}$ Disponível em: http://brincandocomarie.com.br/
} 
VIII Congresso Brasileiro de Informática na Educação (CBIE 2019)

Anais do XXV Workshop de Informática na Escola (WIE 2019)

Contudo, segundo Gil (2012, p. 8) para que o "conhecimento possa ser considerado científico, torna-se necessário identificar operações mentais e técnicas que possibilitam a sua verificação". Diante disto, foi utilizada como método de pesquisa abordagem qualitativa com ênfase na observação participante como técnica de coleta de dados, tendo em vista a faixa etária dos estudantes e o ambiente natural investigado. Neste contexto [Creswell 2010, p. 26] define a pesquisa qualitativa como "um meio para explorar e entender o significado que os indivíduos ou grupos atribuem a um problema social ou humano". Segundo [Lüdke e André 2013, p. 31] a "observação permite a coleta de dados em situações em que é impossível outras formas de comunicação". Para isso, foram executadas as seguintes etapas: (1) seleção dos jogos educativos; (2) aplicação dos jogos selecionados em ambiente real; (3) observação da aplicação e coleta dos dados; e (4) análise dos dados coletados, conforme diagrama da Figura 1.

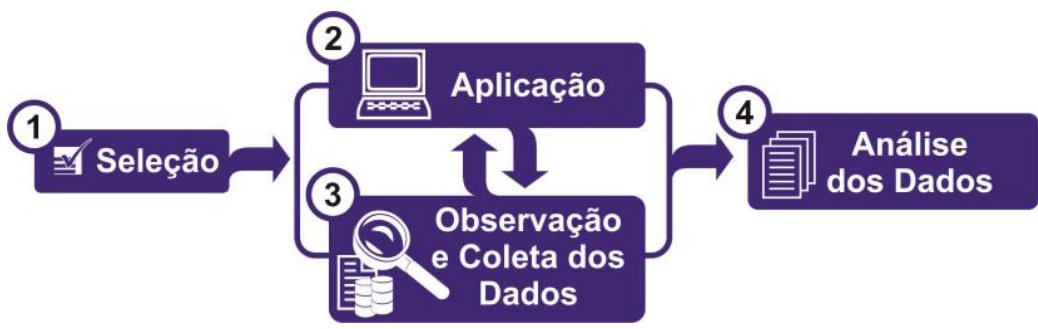

Figura 1. Etapas de execução do estudo.

(Fonte: elaborado pelos autores)

$\mathrm{Na}$ primeira etapa, foram selecionados os jogos educativos para auxiliar no processo de alfabetização de estudantes da Educação Básica, com base na faixa etária entre 6 e 7 anos de idade, design do jogo, usabilidade, interação com usuário e com proposta pedagógica na alfabetização (Língua Portuguesa) nos dois primeiros anos do Ensino Fundamental I, com foco em proporcionar oportunidades de desenvolvimento de acordo com a BNCC em "habilidades de leitura e de escrita e ao seu envolvimento em práticas diversificadas de letramentos" [Brasil 2018a, p. 59]. Como resultado, foram escolhidos: (i) "Capitão Alberto e o Tesouro do Faraó" e (ii) "Chapeuzinho e o Enigma da Floresta", ambos gratuitos e disponíveis na plataforma web. Na segunda etapa, foram executadas atividades disponíveis nos softwares educativos, conforme mostrado na Tabela 2.

Tabela 2. Jogos Digitais Educativos.

\begin{tabular}{|c|c|c|c|}
\hline Plataforma & Jogos Digitais & Breve descrição & Objetivos \\
\hline 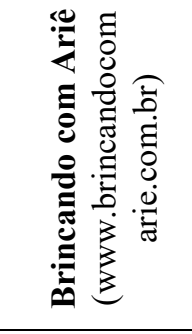 & $\begin{array}{l}\text { Capitão Alberto e c } \\
\text { Tesouro do Faraó } \\
\text { Can }\end{array}$ & $\begin{array}{l}\text { Neste jogo, o Capitão Alberto está no Egito } \\
\text { em busca do tesouro na tumba do Faraó. } \\
\text { Para isso, o estudante precisa clicar ou } \\
\text { digitar a primeira letra referente ao nome de } \\
\text { cada figura para decifrar os enigmas escritos } \\
\text { nas paredes. }\end{array}$ & 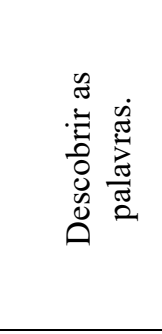 \\
\hline
\end{tabular}




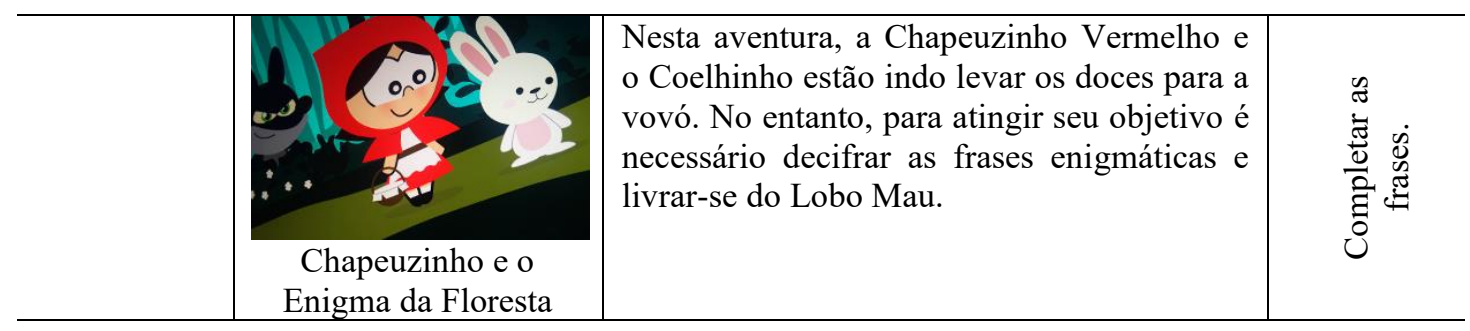

As atividades foram executadas no período de fevereiro e março de 2019. Os estudantes tiveram uma hora de aula por semana no laboratório de informática com a presença dos professores. A priori, a plataforma "Brincando com Ariê" foi acessada e proposta como primeira atividade: o jogo educativo "Capitão Alberto e o Tesouro do Faraó", em seguida, os estudantes foram orientados sobre o funcionamento e objetivo do jogo. Por último, foi usado o jogo "Chapeuzinho e o Enigma da Floresta" com objetivo de completar as frases.

A execução da terceira etapa ocorreu simultaneamente com a segunda, durante o período de aplicação dos jogos educativos foram feitas anotações em um diário de bordo do pesquisador com as observações in loco, fotos, informações descritivas, as falas e comportamentos dos estudantes com o uso dos jogos educativos no laboratório de informática com o suporte docente para auxiliar no processo de desenvolvimento cognitivo, seguindo o modelo para condução de estudos qualitativos propostos por [Creswell 2010; Gil 2012; Lüdke e André 2013]. De acordo com [Lüdke e André 2013, p. 38] "essas medidas têm um caráter meramente prático, no sentido de ajudar a organização e análise dos dados". Na quarta etapa foi realizada a análise dos dados. Na próxima seção, apresentamos os principais resultados e discussão.

\section{Resultados e Discussão}

Ao observar as interações dos estudantes com o primeiro jogo digital "Capitão Alberto e o Tesouro do Faraó" no nível fácil, notou-se que os mesmos se sentiram desafiados para decifrar o enigma, conhecer a palavra escondida na parede, ganhar estrelas no jogo e para avançar de nível. Para isso, faz-se necessário digitar ou clicar na primeira letra de cada figura apresentada no cenário do jogo e muitos estudantes não estavam familiarizados com alguns objetos propostos (conforme mostrado na Figura 2) e começaram a interagir/dialogar entre eles no laboratório de informática para saber $o$ nome do objeto. Comumente, observou-se os estudantes apontando com o dedo na tela do computador e perguntando para o colega ao lado: "ei, você sabe o que isso aqui?", "qual o nome dessa figura?", "o que é isso?" e os colegas interagiram auxiliando um ao outro na tentativa de acerta o nome da figura, criando uma maior interação entre os estudantes durante a realização da atividade e desenvolvendo o trabalho colaborativo para descobrir as apalavras secretas. Interações semelhantes são apontadas por [Machado, Warpechowski e Vaz 2017] o que possibilitou um engajamento na construção colaborativa do conhecimento com o uso da tecnologia.

Em determinados momentos, alguns estudantes já logo perguntaram para a professora: "tia, tia, qual o nome dessa figura?", "tia o que é isso?". Em casos como esse, a professora sempre buscou interagir, auxiliando os estudantes para descobrir o nome da figura e sempre que necessário, explicando a aplicação do mesmo em cenário do mundo real, como por exemplo, na Figura 2, para decifrar o enigma é necessário digitar a letra "A" do objeto "anzol", neste caso a professora, explicou para turma: " $O$ 
VIII Congresso Brasileiro de Informática na Educação (CBIE 2019)

Anais do XXV Workshop de Informática na Escola (WIE 2019)

anzol é uma ferramenta/objeto utilizado pelo pescador para capturar/pegar o peixe". O mesmo aconteceu com palavras que a letra inicial tem som de vogal, mas escreve com consoante, como "helicóptero" mostrado na Figura 2. Alguns estudantes confundiram o nome do objeto (produto) com a marca, como no exemplo da Figura 2, nível difícil "iogurte". Neste cenário, o uso do jogo digital proporcionou diálogos entre os estudantes e professores em ambiente real, criando assim, segundo Valente $(1998$, p. 7) condições de aprendizagem, onde o professor passa a "ser criador de ambientes de aprendizagem e o facilitador do processo de desenvolvimento intelectual do aluno".

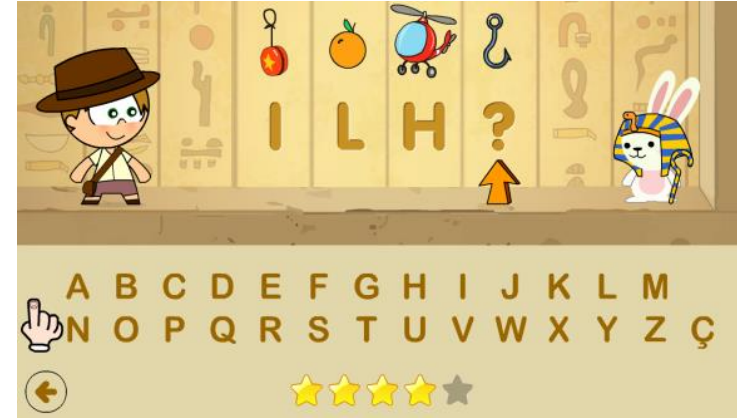

Fácil

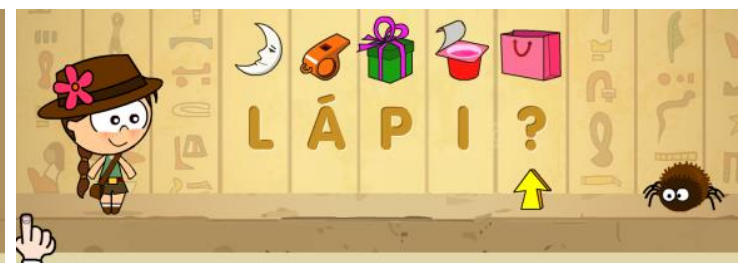

A B C DEF G H I J K L M N O $P$ P $Q R$ R S T T U V W X Y Z C C

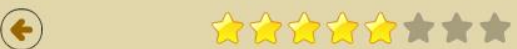

Difícil

Figura 2. Apresentação dos dois níveis do jogo digital educativo "Capitão Alberto e o Tesouro do Faraó". [Brincando com Ariê 2019].

No segundo jogo digital "Chapeuzinho e o Enigma da Floresta”, os estudantes foram desafiados a construir frases. A priori, no nível fácil os estudantes completaram as frases substituindo os objetos pelos seus respectivos nomes em cada frase. No nível difícil, os estudantes completaram as frases substituindo o ponto de interrogação (?) pela a palavra correta. Para isso, no nível fácil, o estudante clicava com o botão direito do mouse e arrastava o respectivo nome para cima do objeto, substituindo o mesmo. Processo semelhante foi realizado no nível difícil, onde o estudante arrastava para cima do ponto de interrogação (?) dentro do jogo digital, conforme mostrado na Figura 3. Nesta fase de construção do conhecimento, notou-se um maior engajamento e motivação dos estudantes na realização das atividades quando comparado com as atividades desenvolvidas sem o uso da tecnologia. Essas observações corroboram com os achados apresentados por Nakano et al. (2018) e contribuem positivamente no processo de aprendizagem com o uso de jogos digitais educativos no contexto da Educação Básica.

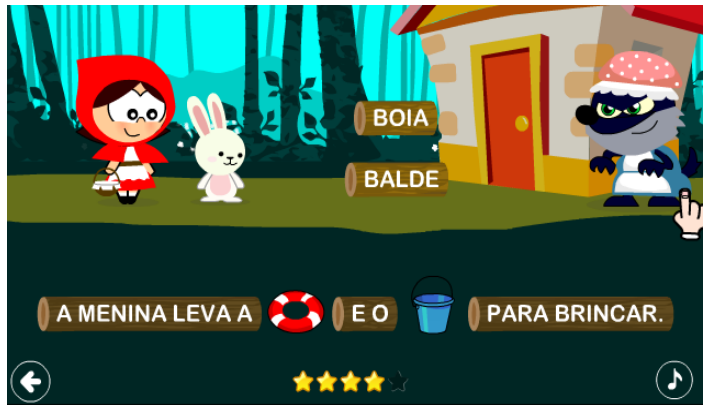

Fácil

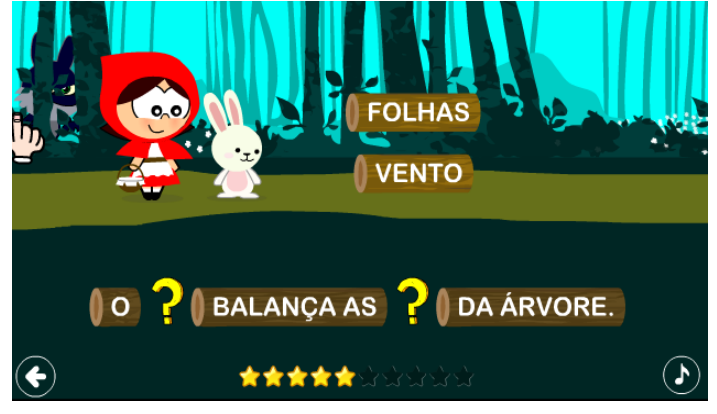

Difícil

Figura 3. Apresentação dos dois níveis do jogo digital educativo "Chapeuzinho e o Enigma da Floresta". [Brincando com Ariê 2019]. 
VIII Congresso Brasileiro de Informática na Educação (CBIE 2019)

Anais do XXV Workshop de Informática na Escola (WIE 2019)

Em vários momentos, observou-se os estudantes realizando a leitura das frases, logo após completa-las junto com os colegas. Em um determinado momento, ao completar corretamente a frase a estudante "A" chamou a professora e mencionou: "tia, tia, olha: o apontador e a caneta estão no estojo". Outros colegas próximos à professora, logo falaram: "veja tia, o sol e a lua ficam no céu”, "tia, olha isso: a bola é do menino”. E isso, despertou o interesse de vários estudantes no laboratório de informática para fazer a leitura em voz alta para a professora e colegas da turma. Resultados semelhantes foram identificados no estudo de Morais et al. (2018, p. 1) ao relatar que o "uso de jogos digitais despertou o interesse e motivação dos alunos em aprender de forma lúdica, interativa e prazerosa, contribuindo para uma aprendizagem significativa".

Além disso, observou-se que alguns estudantes com mais familiaridades com o computador auxiliaram a professora no início das aulas para acessar os jogos digitais online através do browser. Neste contexto, Ramos e Segundo (2018, p. 547) ressaltam "a importância do uso dos jogos digitais com o objetivo de aprimorar as funções cognitivas". Diante disso, notou que o uso dos jogos digitais "Capitão Alberto e o Tesouro do Faraó" e "Chapeuzinho e o Enigma da Floresta", alinhados aos objetivos didático-pedagógicos contribuiu positivamente para aumentar a motivação, engajamento, colaboração entre os estudantes nos processos de ensino e aprendizagem no contexto dos anos iniciais do Ensino Fundamental I.

\section{Considerações Finais}

O presente estudo relatou o uso de jogos digitais educativos como ferramentas para auxiliar no processo de alfabetização de estudantes do Ensino Fundamental I. O uso dos jogos despertou a atenção dos estudantes e possibilitou o aumento da motivação, além da colaboração entre os estudantes e docentes no processo de alfabetização dos estudantes, evidenciando que a seleção, planejamento e a execução adequada dos jogos com foco nos processos de ensino e aprendizagem, podem impactar significativamente o desempenho de aprendizagem dos estudantes. Como trabalhos futuros, objetiva-se contribuir na implementação de novos recursos educacionais digitais para melhorar aprendizagem dos estudantes do Ensino Fundamental, com foco na resolução de problemas (Matemática) e em leitura e interpretação de textos (Língua Portuguesa), bem como, conduzir avaliações experimentais, visando identificar e extrair práticas pedagógicas inovadoras do uso das TDIC em cenário do mundo real para potencializar os processos de ensino e de aprendizagem de docentes e estudantes no contexto da Educação Básica brasileira.

\section{Referências}

Bergamasco, E. C., \& Bergamasco, L. C. C. (2013). A utilização das Tecnologias de Informação e Comunicação na Educação Infantil: avanços e desafios. In Anais do Workshop de Informática na Escola (Vol. 1, No. 1, p. 329).

Bordin, A., \& Quepfert, W. (2018). Projeto de ensino de programação para alunos de ensino médio: Uma análise do cenário e das percepções das oportunidades. In Anais do Workshop de Informática na Escola (Vol. 24, No. 1, p. 205).

Brasil, Ministério da Ciência, Tecnologia, Inovações e Comunicações. (2018b). Estratégia Brasileira para a Transformação Digital. Brasília: MCTIC. 
VIII Congresso Brasileiro de Informática na Educação (CBIE 2019)

Anais do XXV Workshop de Informática na Escola (WIE 2019)

Brasil, Ministério da Educação. (2018a). Base Nacional Comum Curricular. Disponível em: http://basenacionalcomum.mec.gov.br/abase/

Brasil, Presidência da República. (2017). Decreto nº 9.099, de 18 de julho de 2017.

Brincando com Ariê. (2019). Disponível em: http://brincandocomarie.com.br/

Creswell, J. W. (2010). Projeto de Pesquisa: métodos qualitativo, quantitativo e misto. $3^{\mathrm{a}}$ ed. - Porto Alegre: Artmed.

Educação Conectada, Brasil. Ministério da Educação. (2017). Portaria $n^{0} 1.602$, de 28 de dezembro de 2017.

Figueiredo, G., Nobre, I., \& Passos, M. L. S. (2015). Tecnologias computacionais na educação: Desafios na prática docente. In Anais do Workshop de Informática na Escola (Vol. 21, No. 1, p. 127).

Gil, A. C. (2012). Métodos e técnicas de pesquisa social. $6^{\text {a }}$ ed. - São Paulo: Atlas.

Inep, M. (2015). Plano Nacional de Educação PNE 2014-2024: Linha de base. Brasília: INEP.

Lüdke, M., \& André, M. E. D. A. (2013). Pesquisa em educação: abordagens qualitativas. $2^{\mathrm{a}}$ ed. - Rio de Janeiro: E.P.U.

Machado, D., Warpechowski, M., \& Vaz, D. (2017). Modelo de Interação Entre Pares (MIP): viabilizando o ensino da informática educativa na Educação Infantil. In Anais do Workshop de Informática na Escola (Vol. 23, No. 1, p. 353).

Morais, E. V. D., de Castro, M. P., dos Santos, U. N. (2018). Revista Tecnologias na Educação, Vol. 25.

Nakano, R., Rosa, S., Rosa, S. S., de Souza, R. C. T., \& Rosa, V. (2018). Integração de Tecnologias Digitais à Linguagem Oral e Escrita: um estudo de caso no Ensino Fundamental I. In Anais do Workshop de Informática na Escola (Vol. 24, No. 1, p. 147).

Neto, M., dos Santos, C. A. M., de Souza, E. E., \& Fonseca, M. G. (2018). Robótica educacional uma ferramenta para ensino de lógica de programação no ensino fundamental. In Anais do Workshop de Informática na Escola (Vol. 24, No. 1, p. 315).

Oliveira, A. (2018). A Arte de Ensinar Matemática com Uso das Tecnologias Digitais. In Anais do Workshop de Informática na Escola (Vol. 24, No. 1, p. 700).

Oliveira, W., \& Silva Junior, C. G. (2015). Pesquisa, Desenvolvimento e Avaliação de um Jogo para o Ensino de Matemática, Baseado no Processo de Virtualização de Jogos. In Anais dos Workshops do Congresso Brasileiro de Informática na Educação (Vol. 4, No. 1, p. 145).

Oliveira, W., Rodrigues, L., Toda, M. A., Palomino, P. T., \& Isotani, S. (2019). Automatic Game Experience Identification in Educational Games. In Brazilian Symposium on Computers in Education (Vol. 30, No. 1).

Pedró, F. (2016). A tecnologia e as transformações da educação. Fundação Santillana.

Ramos, D. K., \& Segundo, F. R. (2018). Jogos Digitais na Escola: aprimorando a atenção e a flexibilidade cognitiva. Educação \& Realidade, 43(2), 531-550. 
VIII Congresso Brasileiro de Informática na Educação (CBIE 2019)

Anais do XXV Workshop de Informática na Escola (WIE 2019)

Ramos, D. K., Anastácio, B. S., da Silva, G. A., Venturieri, C., Stange, N., Martins, M. E. (2018). Jogos digitais, habilidades cognitivas e motivação: percepção das crianças no contexto escolar. Proceedings of SBGames.

Santana, S. J., Paiva, R., Bittencourt, I. I., Ospina, P. E., de Amorim Silva, R., \& Isotani, S. (2016). Evaluating the impact of Mars and Venus Effect on the use of an Adaptive Learning Technology for Portuguese and Mathematics. In Advanced Learning Technologies (ICALT), 2016 IEEE 16th International Conference on (pp. 31-35). IEEE.

Santana, S., \& Santos, W. O. (2018a). Softwares Educacionais como Auxílio ao Processo de Alfabetização de Estudantes da Educação Infantil. In Anais do Workshop de Informática na Escola (Vol. 24, No. 1, p. 1).

Santana, S., \& Santos, W. O. (2018b). Jogos Educativos no Ensino de Matemática: Qual a Melhor Abordagem? In Anais do Workshop de Informática na Escola (Vol. 24, No. 1, p. 80).

Santana, S., Bittencourt, I. I., de Amorim Silva, R., \& Ospina, P. (2018). Extração e Recomendação de Boas e Más Práticas Pedagógicas a Partir de Processos de Ensino e Aprendizagem usando um Sistema Tutor Inteligente Gamificado. In Simpósio Brasileiro de Informática na Educação (Vol. 29, No. 1, p. 1353).

Santos, J., Melo, M., Serey, D., de Figueiredo, J. C. A., \& Minervino, C. (2018). Análise de um Jogo Educacional para Mensuração de Habilidades Cognitivas Preditoras do Desenvolvimento de Leitura em Crianças. In Anais do Workshop de Informática na Escola (Vol. 24, No. 1, p. 643).

Santos, W. O., \& da Silva Junior, C. G. (2016). Virtualização de Jogos Educativos: Uma Experiência no Ensino de Matemática. Revista Brasileira de Informática na Educação, 24(2).

Santos, W. O., \& Isotani, S. (2018) Desenvolvimento de Jogos Educativos? Desafios, Oportunidades e Direcionamentos de Pesquisa. RENOTE, 16(2).

Santos, W. O., Rosalino, G., \& Silva, C. (2017). Desafios das Diagonais: Um Jogo Casual para o Aprimoramento do Raciocínio Lógico. In Anais dos Workshops do Congresso Brasileiro de Informática na Educação (Vol. 6, No. 1, p. 168).

Sousa Pires, F. G., Michel, F., Bernardo, J. R. S., Melo, R., \& de Freitas, R. (2018). O livro do conhecimento: Um jogo de aventura para exercitar a ortografia. In Simpósio Brasileiro de Informática na Educação (Vol. 29, No. 1, p. 695).

Unesco, Organização das Nações Unidas para a Educação, a Ciência e a Cultura. (2017). TIC, educação e desenvolvimento social na América Latina e o Caribe. Montevidéu, Uruguai.

Valente, J. A. (1998). Computadores e conhecimento: repensando a educação. 2. ed. Campinas, SP: UNICAMP/NIED.

Vidotto, K. N. S., Lopes, L., Pozzebon, E., \& Frigo, L. (2017). Ambiente Inteligente de Aprendizagem MAZK com alunos do Ensino Fundamental II na disciplina de Ciências. In Simpósio Brasileiro de Informática na Educação (Vol. 28, No. 1, p. 1367). 